\title{
Association between ezrin protein expression and the prognosis of colorectal adenocarcinoma
}

\author{
LI-JUAN LIN ${ }^{1}$ and LI-TIAN CHEN ${ }^{2}$ \\ ${ }^{1}$ Department of Medical Imaging, Eastern Liaoning University School of Medicine, Dandong 118002; \\ ${ }^{2}$ Department of Liver Transplantation Surgery, Xin Hua Hospital, Shanghai Jiao Tong \\ University School of Medicine,Shanghai 200092, P.R. China
}

Received January 13, 2013; Accepted April 29, 2013

DOI: $10.3892 / \mathrm{mmr} .2013 .1490$

\begin{abstract}
Ezrin is involved in maintaining cell structure and cell motility. Expression levels of the ezrin gene correlate with numerous human malignancies. The aim of this study was to explore the role of ezrin in tumor progression and the prognostic evaluation of colorectal adenocarcinoma (CRA). The levels of ezrin protein in 186 CRA samples were evaluated using immunohistochemistry. Furthermore, the correlation between the expression of ezrin and the clinicopathological features of CRA was evaluated with the $\chi^{2}$ and Fisher's exact tests, survival rates were calculated using the Kaplan-Meier method, and the correlation between prognostic factors and patient survival was calculated by Cox analysis. Ezrin protein expression demonstrated an immunohistochemical cytoplasmic staining pattern in CRA. The difference between the positive rate of ezrin expression in CRA $(38.7 \%, 72 / 186)$ and the adjacent normal mucosal tissues was deemed to be statistically significant $(91.9 \%, 171 / 186 ; \mathrm{P}=0.000)$. The positive rate of ezrin expression in cases with a large tumor, serosal invasion, lymph node (LN) metastasis, high LN ratio (LNR) and at a late tumor stage was significantly lower than in cases without these factors $(\mathrm{P}=0.044, \mathrm{P}=0.032, \mathrm{P}=0.002, \mathrm{P}=0.011$ and $\mathrm{P}=0.000$, respectively). The 5-year survival rate of $\mathrm{CRA}$ without ezrin expression was lower than CRA with expression $(\mathrm{P}=0.000)$. Furthermore, analysis by Kaplan-Meier demonstrated that CRA cases with poor differentiation, serosal invasion and at a late tumor stage combined with no ezrin expression had a lower survival rate than cases that had these factors plus ezrin expression ( $\mathrm{P}=0.000$, respectively). Additionally, the non-expression of ezrin emerged as a significant independent prognostic factor in CRA prognosis (HR, 0.562; 95\% CI, 0.404-0.783; $\mathrm{P}=0.001$ ),
\end{abstract}

Correspondence to: Dr Li-Tian Chen, Department of Liver Transplantation Surgery, Xin Hua Hospital, Shanghai Jiao Tong University School of Medicine, 1555 Kongjiang Street, Shanghai 200092, P.R. China

E-mail: litianchen669@126.com

Key words: ezrin, colorectal adenocarcinoma, immunohistochemistry, survival analysis in addition to the LNR (HR, 0.589; 95\% CI, 0.369-0.939; $\mathrm{P}=0.026)$ and tumor stage $(\mathrm{HR}, 0.655 ; 95 \% \mathrm{CI}, 0.487-0.880$; $\mathrm{P}=0.005)$. This study demonstrated that ezrin may be useful to identify at-risk patients who may benefit from a more aggressive adjuvant therapy following tumor resection. Ezrin may serve as a useful therapeutic biomarker.

\section{Introduction}

Colorectal adenocarcinoma (CRA) is a common type of malignant tumor. The 5-year survival rate of CRA is $\sim 65 \%$, and the tumor stage, lymph node (LN) status, tumor grade and lymphatic and venous invasion are critical morphological prognostic factors $(1,2)$. Although advances have been made while studying the molecular basis of this disease, the spectrum of genes that reveal altered expression in CRA, as well as their role in the disease, remain unclear $(3,4)$. Therefore, more sensitive CRA biomarkers that are capable of predicting prognosis and guiding effective targeting therapy are required.

The ezrin gene is a member of the ezrin-radixin-moesin (ERM) cytoskeleton-associated protein family and is involved in a wide variety of cellular processes. It is one of the components of cell-surface structures involved in cell adhesion to the extracellular matrix, and has been implicated in membrane-cytoskeleton interactions $(5,6)$. The ezrin protein correlates with tumor invasiveness, metastasis and clinical prognosis in numerous types of human cancer, including CRA (7-9). The molecular characteristics of the ezrin protein may be important during tumor progression (10); however, the clinical significance of these characteristics in human cancer requires clarification. The aim of the present study was to investigate the correlation between ezrin expression, clinicopathological parameters and the prognosis of CRA patients. Our data suggested that cytoplasmic ezrin expression is associated with tumor metastasis and poor survival in CRA patients, and may be a useful marker for predicting disease progression and prognosis in CRA patients.

\section{Materials and methods}

Samples. In total, 186 cases of routinely processed and diagnosed CRA with strict follow-up were randomly selected 
from patients who underwent surgery between 2004 and 2007 in the Liaoning and Shanghai regions of China. Pathological parameters, including age, gender, grade, the presence of nodal metastasis, clinical stage and survival data, were carefully reviewed in all cases. The ages of the patients ranged from 25-82 years, with a mean age of 59.35 years. The male:female ratio was 119:67. Staging was performed according to the TNM and the International Federation of Gynecology and Obstetrics (FIGO) classifications of colonic and rectal carcinomas; according to FIGO, 97 cases were classified as stages I-IIA (early stage). According to the Union for International Cancer Control (UICC) criteria 7th Edition and the WHO classification (Pathology and Genetics of Tumors of the Digestive System), 89 cases were classified as stages IIB-IV (advanced stage). In addition, 99 cases were defined as well-differentiated and 87 cases were defined as moderately or poorly differentiated. All adjacent normal colorectal mucosal tissues from the cancer resection margin were included and none of the patients had received chemotherapy prior to surgery. All 186 patients were followed-up for 5 years or until they succumbed to the disease. At the end of follow-up, 139 patients had survived. Informed consent was obtained from each patient prior to commencing the study and the research protocols were approved by the Ethics Committee of the University Hospitals.

Immunohistochemistry for ezrin in paraffin-embedded tissues. Tissue sections $(4 \mu \mathrm{m})$ were prepared on silane-coated slides (Sigma, St. Louis, MO, USA). Immunostaining kits were purchased from DakoCytomaton Inc. (Glostrup, Denmark) and Nichirei Inc. (Tokyo, Japan). Tissue sections were deparaffinized, rehydrated and incubated with $3 \% \mathrm{H}_{2} \mathrm{O}_{2}$ in methanol for $15 \mathrm{~min}$ at room temperature in order to eliminate endogenous peroxidase activity. The antigen was retrieved at $95^{\circ} \mathrm{C}$ for $20 \mathrm{~min}$ by placing the slides into a $0.01 \mathrm{M}$ sodium citrate buffer $(\mathrm{pH}$ 6.0). The slides were subsequently incubated with a primary ezrin antibody (1:50, BD Biosciences Pharmingen, San Diego, CA, USA) at $4^{\circ} \mathrm{C}$ overnight. Following incubation at room temperature for 30 min with a biotinylated secondary antibody, the slides were incubated with a streptavidin-peroxidase complex (BD Biosciences Pharmingen) at room temperature for $30 \mathrm{~min}$. Immunostaining was developed using chromogen, 3,3'-diaminobenzidine and counterstained with Mayer's hematoxylin. We used mouse IgG isotope controls, which demonstrated negative staining. Furthermore, the positive tissue sections were processed omitting the primary antibody (mouse antiezrin) as negative controls.

Analysis and interpretation of staining. Immunoreactivity was independently evaluated by two researchers who were blinded to the patient outcome. The evaluation was based on the extent and intensity of the staining (12). The ezrin staining intensity was scored as follows: 0 , negative; 1 , weak; 2 , moderate; and 3, strong. Staining extent was scored as follows: $0,0 \% ; 1,1-25 \% ; 2,26-50 \% ; 3,51-75 \%$; and 4 , $76-100 \%$, depending on the percentage of positively stained cells. The sum of the staining intensity and the staining extent scores was used as the final staining score. The specimens were divided into three groups according to their 5-year scores: 0-1, negative (-); 2-4, weakly positive (+); and 5-7, strongly positive $(++)$.

Statistical analysis. Statistical analyses were performed using SPSS 17.0 (SPSS Inc., Chiacgo, IL, USA). The correlation between ezrin expression and clinicopathological characteristics was evaluated using the $\chi^{2}$ and Fisher's exact tests. The survival rates following tumor removal were calculated using the Kaplan-Meier method and the difference in survival curves was analyzed using the log-rank test. Multivariate survival analysis was performed on all significant characteristics and was measured by univariate survival analysis with the Cox proportional hazard regression model. $\mathrm{P}<0.05$ was considered to indicate a statistically significant difference.

\section{Results}

Expression of ezrin protein in CRA. The expression of ezrin protein revealed an immunohistochemical cytoplasmic staining pattern in CRA. The difference between the strongly positive rate $(++)$ in the CRA $(15.6 \%, 29 / 186)$ and adjacent normal mucosal tissues $(25.3 \%, 47 / 186)$ was not deemed to be statistically significant $(\mathrm{P}=0.083)$; however, the positive $(+$ and ++$)$ rate in CRA $(61.3 \%, 114 / 186)$ was significantly lower than that found in the adjacent normal mucosal tissues (91.9\%, 171/186; P=0.000; Table I and Fig. 1)

Clinicopathological and prognostic significance of ezrin expression. To evaluate the role of ezrin protein in CRA progression, we analyzed the correlation between the expression of ezrin protein and major clinicopathological features. Table II and Fig. 3 demonstrate that the positive rate of ezrin expression in cases with a large tumor was $52.1 \%$ (37/71), which was significantly lower than in cases with a smaller tumor $(67.0 \%, 77 / 115 ; \mathrm{P}=0.044)$. The positive rates of ezrin expression in cases without serosal invasion $(68.3 \%, 69 / 101)$ or LN metastasis $(70.3 \%, 78 / 111)$ were significantly higher than in CRA cases with these factors $(\mathrm{P}=0.032$ and $\mathrm{P}=0.002$, respectively). The lymph node ratio (LNR) refers to the examination of the positive $\mathrm{LN}$ rate, and ezrin expression was identified to statistically correlate with the LNR. Cases with a high LNR $(\geq 0.7)$ had a lower positive rate of ezrin expression $(36.4 \%, 8 / 22)$ than cases with a low LNR $(<0.7 ; 64.6 \%, 106 / 164 ; \mathrm{P}=0.011)$. Finally, the positive rate of ezrin expression was $47.2 \%(42 / 89)$ in late-stage CRA, which was significantly lower than in early-stage cases (74.2\%, 72/97; $\mathrm{P}=0.000)$. However, the differences among ezrin expression, age, gender and tumor grade were not statistically significant $(\mathrm{P}>0.05$, respectively).

Ezrin expression level combined with tumor grade, serosal invasion status and stage affects the prognosis of CRA. To confirm the role of ezrin expression in CRA progression, we analyzed the 5-year survival rate of 186 CRA patients using the Kaplan-Meier method and discovered that CRA patients with no ezrin expression had a lower 5-year survival rate than those with positive ezrin expression ( $\mathrm{P}=0.000$; Fig. 2). Furthermore, we analyzed the correlation between other factors (age, gender, tumor size, grade, serosal invasion, LN, LNR and tumor stage) and the 5-year survival rate in CRA 
Table I. Ezrin protein expression in CRA.

\begin{tabular}{|c|c|c|c|c|c|c|}
\hline \multirow[b]{2}{*}{ Diagnosis } & \multirow[b]{2}{*}{ No. } & \multicolumn{3}{|c|}{ Ezrin (n) } & \multirow[b]{2}{*}{ Positive rate $(\%)$} & \multirow[b]{2}{*}{ Strongly positive rate $(\%)$} \\
\hline & & - & + & ++ & & \\
\hline Carcinoma & 186 & 72 & 85 & 29 & $61.3^{\mathrm{a}}$ & 15.6 \\
\hline Normal mucosa & 186 & 15 & 124 & 47 & 91.9 & 26.3 \\
\hline
\end{tabular}

${ }^{a} \mathrm{P}<0.01$, compared with the normal mucosa group. CRA, colorectal adenocarcinoma.

Table II. Univariate analysis of ezrin protein expression and various risk factors in 186 patients with colorectal adenocarcinoma.

\begin{tabular}{|c|c|c|c|c|c|}
\hline \multirow[b]{2}{*}{ Characteristic } & \multirow[b]{2}{*}{ No. } & \multicolumn{2}{|c|}{ Ezrin expression, n (\%) } & \multirow[b]{2}{*}{ OR $(95 \% \mathrm{CI})$} & \multirow[b]{2}{*}{ P-value } \\
\hline & & - & + and ++ & & \\
\hline Age (years) & & & & $0.230(0.680-2.227)$ & 0.297 \\
\hline$>59$ & 82 & $34(41.5)$ & $48(58.5)$ & & \\
\hline$\leq 59$ & 104 & $38(36.5)$ & $66(63.5)$ & & \\
\hline Gender & & & & $0.901(0.488-1.663)$ & 0.429 \\
\hline Male & 119 & $45(37.8)$ & $74(62.2)$ & & \\
\hline Female & 67 & $27(40.3)$ & $40(59.7)$ & & \\
\hline Tumor grade & & & & $0.585(0.323-1.059)$ & 0.057 \\
\hline Well & 99 & $32(32.3)$ & $67(67.7)$ & & \\
\hline Moderate and poor & 87 & $40(46.0)$ & $47(54.0)$ & & \\
\hline Tumor size & & & & $0.537(0.293-0.985)$ & $0.044^{\mathrm{a}}$ \\
\hline$\leq 5 \mathrm{~cm}$ & 115 & $38(33.0)$ & $77(67.0)$ & & \\
\hline$>5 \mathrm{~cm}$ & 71 & $34(47.9)$ & $37(52.1)$ & & \\
\hline Serosal invasion & & & & $1.917(1.054-3.484)$ & $0.032^{\mathrm{a}}$ \\
\hline Present & 85 & $40(47.1)$ & $45(52.9)$ & & \\
\hline Absent & 101 & $32(31.7)$ & $69(68.3)$ & & \\
\hline LN metastasis & & & & $2.561(1.393-4.708)$ & $0.002^{\mathrm{b}}$ \\
\hline Present & 75 & $39(52.0)$ & $36(48.0)$ & & \\
\hline Absent & 111 & $33(29.7)$ & $78(70.3)$ & & \\
\hline LNR & & & & $3.198(1.267-8.072)$ & $0.011^{\mathrm{a}}$ \\
\hline$\geq 0.7$ & 22 & $14(63.6)$ & $8(36.4)$ & & \\
\hline$<0.7$ & 164 & $58(35.4)$ & $106(64.6)$ & & \\
\hline Clinical stage & & & & $3.223(1.740-5.971)$ & $0.000^{\mathrm{b}}$ \\
\hline I-II & 97 & $25(25.8)$ & $72(74.2)$ & & \\
\hline IIIa-IV & 89 & $47(52.8)$ & $42(47.2)$ & & \\
\hline
\end{tabular}

LN, lymph node; LNR, lymph node rate. ${ }^{\mathrm{a}} \mathrm{P}<0.05,{ }^{\mathrm{b}} \mathrm{P}<0.01$.

and discovered that tumor grade, LNR, serosal invasion and tumor stage were key factors associated with 5-year survival rate $(\mathrm{P}=0.016, \mathrm{P}=0.004, \mathrm{P}=0.015$ and $\mathrm{P}=0.006$, respectively). Further combination analysis revealed that CRA with poor differentiation combined with no ezrin expression had the lowest 5-year survival rate, and this was significantly lower than that in cases with ezrin expression (Fig. 3A; $\mathrm{P}=0.000$ ). Furthermore, CRA without serosal invasion combined with positive ezrin expression had the highest 5-year survival rate, and CRA with serosal invasion combined with no ezrin expression had a lower 5-year survival rate than cases with a positive level of ezrin expression (Fig. 3B; $\mathrm{P}=0.000$ ). The 5-year survival rate of CRA patients with a high LNR did not correlate with the cases, whether it was accompanied by ezrin expression or not (Fig. 3C; $\mathrm{P}=0.156$ ). However, the 5-year survival rate of CRA patients with a low LNR did correlate with the cases, whether it was accompanied by ezrin expression or not (Fig. 3C; $\mathrm{P}=0.008$ ). Tumor stage is the most important histological prognostic factor in CRA (11) and we revealed that CRA patients with a high stage (IIIa-IV) and no 
A

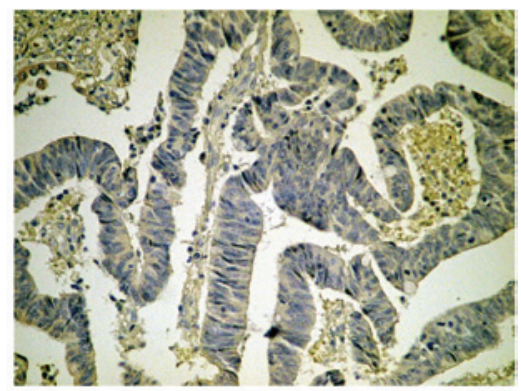

B

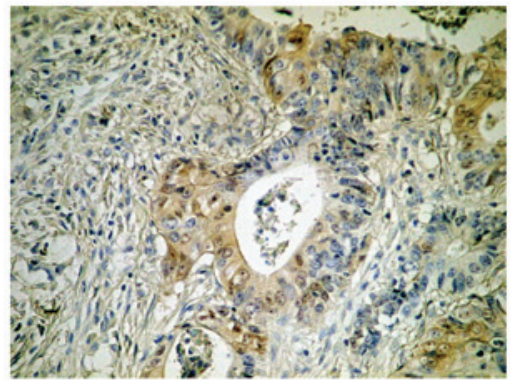

C

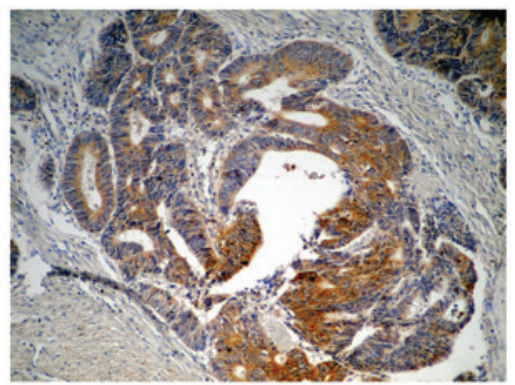

D

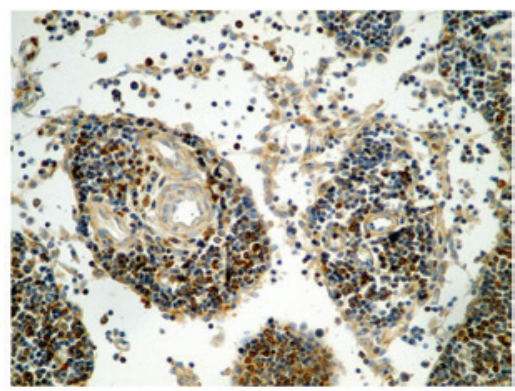

Figure 1. Immunohistochemical staining of ezrin in colorectal adenocarcinoma (CRA). (A) CRA with no ezrin expression; (B) CRA with weak ezrin expression; (C) CRA with marked ezrin expression; (D) Ezrin is not expressed in metastatic lymph nodes, but is potently expressed in the adjacent normal lymph node tissue. (Original magnification, x100).

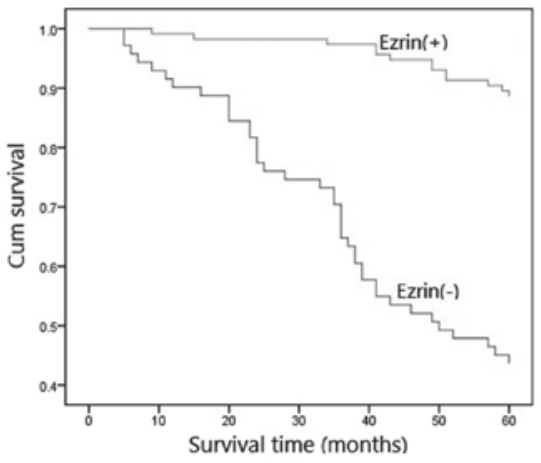

Figure 2. Kaplan-Meier analysis of the overall survival rate in 186 CRA patients in relation to ezrin protein expression. Patients with no ezrin expression had a significantly lower 5-year survival rate $(\mathrm{P}=0.000) .+$, positive; -, negative; CRA, colorectal adenocarcinoma.
$\mathbf{A}$

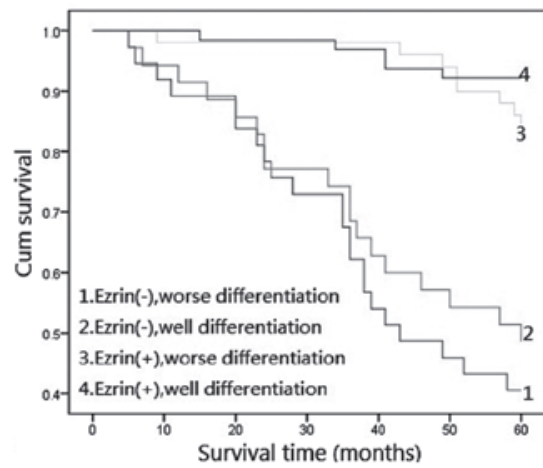

B

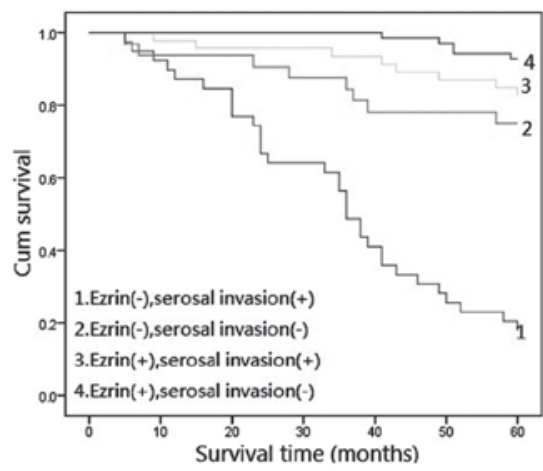

$\mathbf{C}$

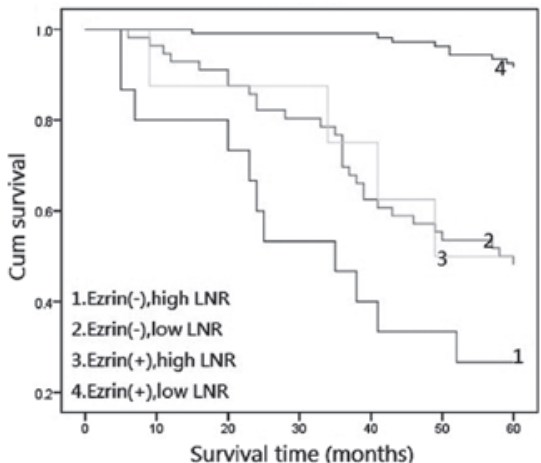

D

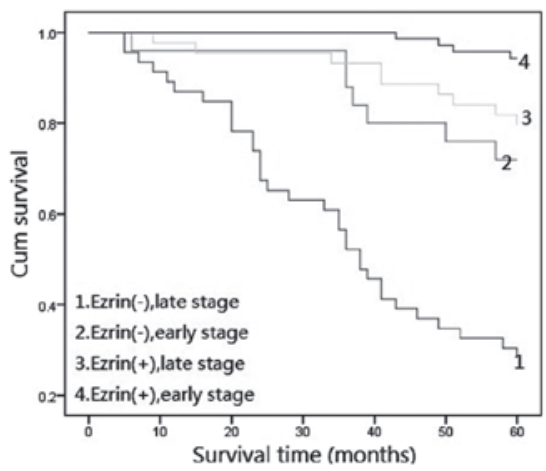

Figure 3. Kaplan-Meier analysis of the overall survival rate in 186 CRA patients with or without ezrin expression in relation to serosal invasion, tumor grade, lymph node ratio (LNR) status and tumor stage. The P-value was calculated by comparing all four groups (log rank test). (A) CRA cases with poor differentiation concomitant with the non-expression of ezrin were associated with the worst 5-year survival rate, significantly worse than CRA with poor differentiation only $(\mathrm{P}=0.000)$. (B) CRA with serosal invasion concomitant with the non-expression of ezrin had the lowest 5-year survival rate and a lower 5-year survival rate than CRA with serosal invasion only $(\mathrm{P}=0.000)$. (C) CRA cases with a high LNR concomitant with the non-expression of ezrin were associated with the worst 5-year survival rate, but not significantly worse than CRA with a high LNR only ( $\mathrm{P}=0.156)$. (D) Late-stage CRA concomitant with the non-expression of ezrin was associated with the worst 5-year survival rate, which was significantly worse than only late-stage CRA $(\mathrm{P}=0.000)$. +, positive; -, negative; CRA, colorectal adenocarcinoma. 
Table III. Univariate and multivariate survival analyses (Cox regression model) of various factors in 186 patients with CRA.

\begin{tabular}{|c|c|c|c|c|c|}
\hline Factor & $\mathrm{B}$ & SE & Wald & HR $(95 \%$ CI $)$ & P-value \\
\hline \multicolumn{6}{|c|}{ Univariate survival analyses } \\
\hline Age (years) & -0.016 & 0.148 & 0.011 & $0.984(0.737-1.315)$ & 0.916 \\
\hline Gender & 0.028 & 0.153 & 0.035 & $1.029(0.763-1.388)$ & 0.852 \\
\hline Tumor size $(\mathrm{cm})$ & -0.156 & 0.151 & 1.068 & $0.856(0.636-1.150)$ & 0.301 \\
\hline Tumor grade & -0.120 & 0.147 & 0.663 & $0.887(0.665-1.183)$ & 0.416 \\
\hline Serosal invasion & -0.038 & 0.149 & 0.065 & $0.963(0.719-1.289)$ & 0.799 \\
\hline LN metastasis & -0.274 & 0.148 & 3.447 & $0.760(0.569-1.015)$ & 0.063 \\
\hline LNR & -0.622 & 0.228 & 7.464 & $0.537(0.344-0.839)$ & $0.006^{\mathrm{b}}$ \\
\hline Clinical stage & -0.445 & 0.147 & 9.127 & $0.641(0.480-0.855)$ & $0.003^{\mathrm{b}}$ \\
\hline Ezrin & -0.605 & 0.152 & 15.901 & $0.546(0.406-0.735)$ & $0.000^{\mathrm{b}}$ \\
\hline \multicolumn{6}{|c|}{ Multivariate survival analyses } \\
\hline Age (years) & 0.043 & 0.153 & 0.078 & $1.043(0.774-1.408)$ & 0.781 \\
\hline Gender & 0.078 & 0.166 & 0.221 & $1.081(0.781-1.498)$ & 0.638 \\
\hline Tumor size (cm) & -0.076 & 0.165 & 0.213 & $0.927(0.671-1.281)$ & 0.644 \\
\hline Tumor grade & -0.115 & 0.150 & 0.580 & $0.892(0.664-1.197)$ & 0.446 \\
\hline Serosal invasion & 0.246 & 0.164 & 2.230 & $1.278(0.926-1.765)$ & 0.135 \\
\hline $\mathrm{LN}$ metastasis & -0.276 & 0.154 & 3.232 & $0.759(0.561-1.025)$ & 0.072 \\
\hline LNR & -0.530 & 0.238 & 4.945 & $0.589(0.369-0.939)$ & $0.026^{\mathrm{a}}$ \\
\hline Clinical stage & -0.424 & 0.151 & 7.862 & $0.655(0.487-0.880)$ & $0.005^{\mathrm{b}}$ \\
\hline Ezrin & -0.576 & 0.169 & 11.628 & $0.562(0.404-0.783)$ & $0.001^{\mathrm{b}}$ \\
\hline
\end{tabular}

CRA, colorectal adenocarcinoma; LN, Lymph node; LNR, Lymph node rate. ${ }^{\mathrm{a}} \mathrm{P}<0.05$, ${ }^{\mathrm{b}} \mathrm{P}<0.01$. B , coefficient; SE, standard error; Wald, Wald statistic; HR, hazard ratio; $\mathrm{CI}$, confidence interval.

ezrin expression had a significantly lower 5-year survival rate than those patients with ezrin expression (Fig. 3D; $\mathrm{P}=0.002$ ). Therefore, the non-expression of ezrin may be a poor prognostic marker for CRA with poor differentiation, serosal invasion, high LNR and at a late stage.

Non-expression of ezrin is an independent prognostic factor in CRA, as determined by the Cox proportional hazard regression model. Table III shows the univariate and multivariate analyses performed using the Cox proportional hazards model. The LNR (HR, 0.589; 95\% CI, 0.369-0.939; $\mathrm{P}=0.026$ ) and tumor stage (HR, 0.655; 95\% CI, 0.487-0.880; $\mathrm{P}=0.005$ ) were demonstrated to be independent prognostic factors for survival in CRA. Notably, the non-expression of ezrin emerged as a significant independent prognostic factor in CRA (HR, 0.562; 95\% CI, 0.404-0.783; P=0.001).

\section{Discussion}

The ezrin gene is located on chromosome 6q25.2-q26. The full length mRNA is $3166 \mathrm{bp}$, encoding a protein that consists of 585 amino acids. In 1981, Fehon et al (5) separated and purified the protein in the small intestinal epithelial cell brush border of a chicken. The ezrin gene belongs to the ERM family and addresses all types of epithelium, shares a homology with the amino terminal membrane-binding domain of erythrocyte band 4.1, and is also involved in membrane-cytoskeleton interactions (13). Ezrin is capable of interacting with several membrane proteins, including CD44, CD43 (14), intercellular adhesion molecule-1, intercellular adhesion molecule-2 and phosphatidylinositol-bisphosphate (15). Ezrin, moesin and radixin are often all expressed on the intramembrane. Ezrin may alter the relationship between the cell membrane and the cytoskeleton and promote the formation of microvilli and aphosphyses, which move cells. The main function of ezrin is to interact with p85, the regulatory subunit of PI3-kinase (PI3K), involved in determining the survival of the epithelial cells by activating the PI3K/Akt pathway (16). Subsequently, the regulation of adhesion, migration and invasion were observed and shown to be important to tumor development and progression (17).

Two theories exist with regard to the role of ezrin in tumor invasion and metastasis. One states that ezrin is the promotion factor for tumor invasion and metastasis and is overexpressed in a variety of invasive carcinoma tissues. In 2004, Yu et al (18) and Khanna et al (19) reported that ezrin is crucial for the metastasis of rhabdomyosarcoma and bone sarcoma in children; they transferred the Vil2 gene (ezrin coding gene) into low-transfer ability cell lines and discovered that the transfer capability of the tumor cell was greatly improved, and the creation of lung metastases was simple. This demonstrates that the overexpression of ezrin in tumor cells may provide the cell with a high transfer activity. Contrary to the upregulation of ezrin in tumor cells, another hypothesis is that ezrin is downregulated in tumor cells and inhibits tumor invasion and metastasis. Karmakar and Das (20) observed that in the human chorioepithelioma cell line JEG-3 cultivated by IL-IB, ezrin, E-cadherin and $\beta 2$ serial protein 
expression levels were downregulated, CD44 expression was enhanced, adhesion between the JEG-3 cells and the matrix was increased, cell-cell adhesion abated and aggressiveness was enhanced. Hiscox and Jiang (21) cultured the colon cancer cell line in vitro and observed that a lack of ezrin decreased tumor cell aggregation, increased the separation phenomenon, broadened the intercellular space, formatted pseudopodia, increased the ability to move, decreased intercellular adhesion, increased matrix adhesion and enhanced aggressiveness. Our study suggests that ezrin expression is deregulated in CRA and that the non-expression of ezrin exerts a profound effect on cellular proliferation, cell adhesion, invasion and aggressiveness during cancer growth and metastasis.

Tumor staging is an important histological feature of CRA prognosis, but knowledge of its associated molecules is very limited. In this study, no ezrin expression was identified in a third of CRA cases, which correlated with the tumor size, serosal invasion status, LNR and the pathological stage. Additionally, the non-expression of ezrin in early-stage CRA was three times lower than in the late-stage cases. Furthermore, the cases had a lower 5-year survival rate, and more notably, late-stage CRA accompanied by ezrin non-expression resulted in a poorer survival rate than that in late-stage CRA only. These results indicate that tumor stage and the non-expression of ezrin may indicate the survival of patients. Additionally, tumor staging was found to be an independent prognostic factor, and we demonstrated that the non-expression of ezrin is associated with the poor prognosis of late-stage CRA.

According to the recommendations of the American Joint Committee on Cancer (AJCC) and the National Cancer Institute, $\geq 12$ LNs should be examined in order to confirm the absence of nodal involvement in colorectal cancer patients $(1,3)$, due to the fact that the number of LNs examined is a reflection of the aggressiveness of the surgical dissection and positive pathological identification (22). Additionally, the number of positive LNs examined in colorectal surgery may be associated with the outcome for the patient. Telian and Bilchik (23) reported that the LNR, rather than LN number, is an important positive prognostic factor for colorectal cancer. Wang et al (24) concluded that LNR is an independent survival predictor after adjusting for the age of the patient, tumor size, tumor grade, ethnicity, number of positive LNs and total number of LNs collected. Therefore, LNR $\geq 12$ may be a better prognostic indicator than the number of positive LNs. Consistent with other reports, we confirmed that a high LNR $(\geq 12)$ is an independent prognostic factor for patient survival in CRA. Moreover, CRA with a low LNR had 3-fold ezrin expression compared with a high LNR in CRA. Additionally, CRA with a decreased LNR without ezrin expression had the lowest 5-year survival rate, even though it was not statistically lower than a high LNR with ezrin expression.

In conclusion, we identified ezrin as a potential biomarker to evaluate the tumor progression and prognosis of CRA. The non-expression of ezrin was more commonly observed in cases with poor CRA prognostic factors, leading to late-stage tumors and short survival times. Ezrin has been suggested as a novel therapeutic method for selectively targeting cancer cells, particularly for late-stage CRA.

\section{References}

1. Zlobec I and Lugli A: Prognostic and predictive factors in colorectal cancer. J Clin Pathol 61: 561-569, 2008.

2. Berman BP, Weisenberger DJ, Aman JF, et al: Regions of focal DNA hypermethylation and long-range hypomethylation in colorectal cancer coincide with nuclear lamina-associated domains. Nat Genet 44: 40-46, 2011.

3. Carlisle J, Swart M, Dawe EJ and Chadwick M: Factors associated with survival after resection of colorectal adenocarcinoma in 314 patients. Br J Anaesth 108: 430-435, 2012.

4. Li D, Yan D, Tang H, Zhou C, et al: IMP3 is a novel prognostic marker that correlates with colon cancer progression and pathogenesis. Ann Surg Oncol 16: 3499-3506, 2009.

5. Fehon RG, McClatchey AI and Bretscher A: Organizing the cell cortex: the role of ERM proteins. Nat Rev Mol Cell Biol 11: 276-287, 2010.

6. Neisch AL and Fehon RG: Ezrin, radixin and moesin: key regulators of membrane-cortex interactions and signaling. Curr Opin Cell Biol 23: 377-382, 2011.

7. Xie JJ, Xu LY, Wu ZY, et al: Prognostic implication of ezrin expression in esophageal squamous cell carcinoma. J Surg Oncol 104: 538-543, 2011.

8. Korkeila EA, Sundström J,Pyrhönen S and Syrjänen K: Carbonic anhydrase IX, hypoxia-inducible factor- $1 \alpha$, ezrin and glucose transporter-1 as predictors of disease outcome in rectal cancer: multivariate Cox survival models following data reduction by principal component analysis of the clinicopathological predictors. Anticancer Res 31: 4529-4535, 2011.

9. Zhang XQ, Chen GP, Wu T, Yan JP and Zhou JY: Expression and clinical significance of ezrin in non-small-cell lung cancer. Clin Lung Cancer 13: 196-204, 2012.

10. Schlecht NF, Brandwein-Gensler M, Smith RV, et al: Cytoplasmic ezrin and moesin correlate with poor survival in head and neck squamous cell carcinoma. Head Neck Pathol 6: 232-243, 2012.

11. O'Connell JB, Maggard MA and Ko CY: Colon cancer survival rates with the new American Joint Committee on Cancer sixth edition staging. J Natl Cancer Inst 96: 1420-1425, 2004.

12. Wu Q, Li Z, Lin H, et al: DEK overexpression in uterine cervical cancers. Pathol Int 58: 378-382, 2008.

13. Takeuchi K, Kawashima A, Nagafuchi A and Tsukita S: Structural diversity of band 4.1 superfamily members. J Cell Sci 107: 1921-1928, 1994.

14. Yonemura S, Hirao M, Doi Y, et al: Ezrin/radixin/moesin (ERM) proteins bind to a positively charged amino acid cluster in the juxta-membrane cytoplasmic domain of CD44, CD43, and ICAM-2. J Cell Biol 140: 885-895, 1998.

15. Heiska L, Alfthan K, Grönholm M, et al: Association of ezrin with intercellular adhesion molecule-1 and -2 (ICAM-1 and ICAM-2). Regulation by phosphatidylinositol 4, 5-bisphosphate. J Biol Chem 273: 21893-21900, 1998.

16. Gautreau A, Poullet P, Louvard D and Arpin M: Ezrin, a plasma membrane-microfilament linker, signals cell survival through the phosphatidylinositol 3-kinase/Akt pathway. Proc Natl Acad Sci USA 96: 7300-7305, 1999.

17. Zhou B, Leng J, Hu M, et al: Ezrin is a key molecule in the metastasis of MOLT4 cells induced by CCL25/CCR9. Leuk Res 34: 769-776, 2010.

18. Yu Y, Khan J, Khanna C, et al: Expression profiling identifies the cytoskeletal organizer ezrin and the developmental homeoprotein Six-1 as key metastatic regulators. Nat Med 2: 175-181, 2004.

19. Khanna C, Wan X, Bose S, et al: The membrane-cytoskeleton linker ezrin is necessary for osteosarcoma metastasis. Nat Med 10: 182-186, 2004.

20. Karmakar S and Das C: Modulation of ezrin and E-cadherin expression by IL-1beta and TGF-beta1 in human trophoblasts. J Reprod Immunol 64: 9-29, 2004.

21. Hiscox S and Jiang WG: Ezrin regulates cell-cell and cell-matrix adhesion, a possible role with E-cadherin/beta-catenin. J Cell Sci 112: 3081-3090, 1999.

22. Gao P, Song YX, Wang ZN, et al: Integrated ratio of metastatic to examined lymph nodes and number of metastatic lymph nodes into the AJCC staging system for colon cancer. PLoS One 7: e35021, 2012.

23. Telian SH and Bilchik AJ: Significance of the lymph node ratio in stage III colon cancer. Ann Surg Oncol 15: 1557-1578, 2008.

24. Wang J, Kulaylat M, Rockette H, et al: Should total number of lymph nodes be used as a quality of care measure for stage III colon cancer? Ann Surg 249: 559-563, 2009. 